\title{
PROTOCOLO OPC SOMADO A SISTEMAS DE CONTROLES INDUSTRIAS DESENVOLVIDOS COM JAVA E SCILAB
}

\author{
BARRoso D. S. ${ }^{1}$, QUINTINO J. A. ${ }^{1}$, GOMES F. J. ${ }^{2}$. \\ 1. Faculdade de Engenharia, Universidade Federal de Juiz de Fora, \\ BairroMartelos, CEP: 36036-330 - Juiz de Fora - MG \\ Emails:dionathan.barroso@engenharia.ufjf.br,jessica.quintino@engenharia \\ .ufjf.br
}
2. Departamento de Energia, Universidade Federal de Juiz de Fora, BairroMartelos, CEP: 36036-330 - Juiz de Fora - MG
E-mails: chico.gomes@ufjf.edu.br

\begin{abstract}
The objective of this work is to present one way of perform the training of professionals of the area Process Control and Automation, through the communication protocol OLE for Process Control (OPC), highlighting their features and an alleged form of use of this protocol. Succinctly, the article will present an introduction, and following, a topic on the OPC protocol, describing some definitions and characteristics. As subsequent topics present a methodology for training, based on three modules laboratory, the last still under development, the results of simulations of the control level of a reservoir, by means of the PID controller, directed to our goals. As a last topic will be made conclusions pertinent to all development, as well as future work.
\end{abstract}

Keywords—Control PID, Programmable Logic Controller, Scilab, JAVA, Protocol OPC.

\begin{abstract}
Resumo- $\mathrm{O}$ objetivo deste trabalho tem como enfoque apresentar uma forma de realizar o treinamento de profissionais da área de Controle de Processos e Automação, através do protocolo de comunicação OLE for Process Control (OPC); evidenciando as suas características e uma suposta forma de utilização deste protocolo. De forma sucinta, o trabalho apresentará uma introdução e, em sequência, um tópico sobre o protocolo OPC, descrevendo algumas definições e características. Como tópicos subsequentes apresentaremos uma metodologia para treinamento, baseada em três módulos laboratoriais, o último ainda em desenvolvimento, os resultados de simulações do controle de nível de um reservatório, por meio do controlador PID, direcionados aos nossos objetivos. Como último tópico serão feitas as conclusões pertinentes sobre todo o desenvolvimento, bem como os trabalhos futuros.
\end{abstract}

Palavras-chave—Controladores PID, Controlador Lógico Programável, Scilab, JAVA, Protocolo OPC.

\section{Introdução}

O desenvolvimento da tecnologia e seu constante aprimoramento permitem com que, atualmente, tenhamos uma indústria diversificada que propicia a criação de novos dispositivos para as mais variadas aplicações. Neste cenário, as indústrias com enfoque em automação desenvolvem softwares para a manipulação de dados obtidos através de seus dispositivos por meio de protocolos de comunicação.

Todavia, em consequência deste fato, há um fator favorável para o surgimento de distintos protocolos de comunicação. Fato que culmina na dificuldade da comunicação entre dispositivos de fabricantes diferentes, como sensores, motores, computadores, etc. Ainda como consequência percebe-se o aumento da complexidade dos sistema devido às necessárias conexões entre os elementos do mesmo sistema, em que a comunicação fator é importantíssimo.

Eis que surge, neste cenário, o protocolo de comunicação OPC desenvolvido para a indústria de automação e suas necessidades de unificação dos protocolos, que especifica um conjunto de regras escritas e procedimentos de modo que múltiplos pro- gramas ou aplicações possam se comunicar uns com os outros (OPC Foundation, 2012).

Esta possibilidade nos concede uma ampla aplicação de controle aos mais variados tipos de problemas, o que associado a potencialidade do MATLAB, SCILAB, e a linguagem de programação JAVA permitem a utilização de técnicas diversas, o que torna grande a aplicabilidade para as indústrias e artifício para a educação em engenharia de controle.

Neste contexto, este trabalho inicialmente propõe uma aplicação do protocolo OPC para o controle de nível através dos controladores P, PI, PID digitais de posição sintonizados, através do Método de Ziegler e Nichols, posteriormente, controles de temperatura, pressão e vazão, controlados via "softwares clientes" em tempo real.

\section{Motivação}

Na busca por soluções que proporcionassem o aprofundamento na aquisição de conhecimento sobre o protocolo OPC, em um meio de garantir a transferência deste aprendizado aos demais discentes e pesquisadores, bem como para o treinamento de profissionais na utilização de técnicas, e pesquisa na área de Controle de Processos. Começou-se então o 
desenvolvimento deste trabalho, foram propostos inicialmente trabalhos de menor porte e que tivesse baixo custo, módulo simples e rústico (módulo 1) para controle de nível, para logo em seguida dar um mais passo e adicionar mais uma variável (módulo 2) para controle de temperatura, e assim, se chegar ao ponto de se trabalhar com um módulo laboratorial robusto (módulo 3) que contém: sensores, atuadores e componentes utilizados no chão de fábrica. Nos tópicos a seguir apresentaremos estes módulos de forma sucinta e objetiva.

\section{Uma breve descrição do OPC}

O protocolo OPC é um protocolo de comunicação desenvolvido diante da necessidade da indústria e automação, que carecer de uma comunicação única entre dispositivos, a fim de reduzir tantos os custos de integração, bem como, o ciclo total para os softwares que na indústria de automação e controle, de modo geral. Logo, este desenvolvimento propiciou a padronização da comunicação de dados através de um conjunto de especificações e regras de escrita e procedimentos (OPC Foundation, 2012).

A arquitetura OPC pode ser classificada em duas hierarquias, em "clientes" OPC e "servidores" OPC. Os clientes OPC são aplicações (softwares) que se conectam com um ou mais servidores para interagir com os itens disponibilizados, efetuando a leitura das informações de entrada e escrita nos itens de saída, quando necessário. Os Servidores (fonte de dados) são softwares disponibilizados pelo fabricante de um CLP, ou demais equipamentos de campo, sendo os computadores os responsáveis pelo reconhecimento de drivers e compartilhamento dos dados, fornecidos pelo servidor OPC, que reconhecem os dados, provenientes da rede de comunicação dos equipamentos da planta industrial, e os "traduzem" para o padrão OPC. (SANTOS NETO, 2012). Essa comunicação é válida somente para OPC-DA (Data Access), uma vez que existem diferentes tecnologias OPC.

\section{Módulos Laboratoriais}

\subsection{Módulo Laboratorial 1-Módulo de Nível}

Este módulo é caracterizado por sua simplicidade, fácil construção e aquisição dos materiais utilizados, por ser portátil e de baixíssimo custo.

O módulo de nível utilizado se compõe dos seguintes elementos:

- 1 sensor de nível

- 2 eletrobombas

- 2 reservatórios

Estes elementos estão expostos da seguinte maneira, como apresentada logo em seguida na figura 1 .

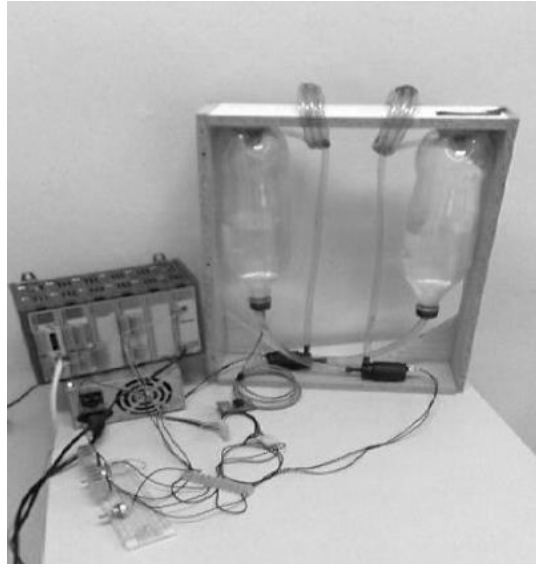

Figura 1. Módulo de Nível

$\mathrm{Na}$ figura 1 acima consegue-se observar o sensor de nível que está acoplado ao reservatório através da mangueira, bem como o seu contraste de proporções quando comparado com o módulo por inteiro; Ainda podemos observar os reservatórios suspensos pelo suporte e as bombas logo abaixo dos mesmos.

Neste sistema proposto e nos demais, entre o módulo de nível e o computador utilizamos um dispositivo chamado CLP.

Os CLP's, ou os Controladores Lógicos Programáveis, foram desenvolvidos no final dos anos 60, com a finalidade de substituir painéis de relés em controles baseados em lógicas combinacional / sequenciais, em linhas de montagem nas indústrias de manufatura, principalmente automobilística, sendo progressivamente adotados pelas indústrias de processos (Atos Automação Industrial, 2006).

De forma simplificada podemos descrever que o cerne do sistema é o protocolo de comunicação OPC, que permite com que o CLP envie e receba valores de tensões ou correntes de origem analógica ou digital, em consequência do equipamento permitir o uso de um servidor OPC. Como o CLP da Atos utilizado não fornece uma corrente necessária para alimentar a bomba foi utilizado um circuito auxiliar, formado por um transistor, um resistor e uma fonte de alimentação, de forma que o CLP controla o transistor como uma fonte de corrente que, consequentemente, controla a bomba ou motor de esguicho.

A técnica de controle elegida para ser aplicada junto aos dados fornecidos pelo servidor OPC foi o controlador PID, por motivo de simplicidade e robustez perante a variados processos.

Normalmente, as implementações utilizando controladores PID envolvem técnicas analógicas. Quando os processos envolvidos utilizam-se de computadores digitais não mais estamos tratando de uma aplicação analógica, pois o processo de amostragem deve, então, ser acrescido à lei de controle (Astrom et al., 1995).

De acordo com o descrito anteriormente, a aplicação deste trabalho também se insere no escopo de um processo de obtenção de dados digital, como exemplo podemos citar que o servidor OPC foi con- 
figurado para adquirir os informações do módulo de nível com um período de $100 \mathrm{~ms}$. Diante destas conclusões a lei de controle não mais será a do PID analógico, tão conhecida academicamente, e sim a lei descrita abaixa obtida por meio da aproximação da regra "backward rectangular" (Astrom et al., 1995) como:

$$
u(k)=K_{p}\left(e(k)+K_{i} i(k)+K_{d} e(k)\right)
$$

Onde $u$ é a variável de controle, $e$ o controle de erro, relativo à referência, o índice $k$ a representação da discretização do processo e a integral do erro; $K_{\mathrm{p}} \mathrm{O}$ ganho proporcional; $K$ i o ganho integral que é a relação do tempo de amostragem divido pelo tempo integral ou $T_{i} ; K_{d}$ o ganho derivativo que é a relação do tempo derivativo, ou $\mathrm{T}_{\mathrm{d}}$, dividido pelo tempo de discretização.

Como consequência do uso do controlador PID há a necessidade da definição dos parâmetros do controle. Devido à grande simplicidade e aplicabilidade do método de Ziegler e Nichols, foi escolhido um dos métodos para a sintonia dos controladores, o Método da Resposta ao Degrau (Ziegler et al., 1942).

Na tabela 1, que seque temos os valores de sintonia dos controladores PID proposto por Ziegler e Nichols.

Tabela 1. Sintonia dos controladores PID segundo Ziegler e Nichols.

\begin{tabular}{|c|c|c|c|}
\hline Controlador & $K_{p}$ & $T_{i}$ & $T_{d}$ \\
\hline $\mathrm{P}$ & $\tau /(k \theta)$ & --- & --- \\
\hline $\mathrm{PI}$ & $0,9 \tau /(k \theta)$ & $3,33 \theta$ & --- \\
\hline $\mathrm{PID}$ & $1,2 \tau /(k \theta)$ & 2 & $0,5 \theta$ \\
\hline
\end{tabular}

Onde:

- $\tau$ é a constante de tempo;

- $\theta$ é o tempo morto;

- $\quad k$ é o ganho do processo, ou relação en-tre a resposta estacionária $\left(y_{s s}\right)$ e o de-grau aplicado.

A metodologia empregada consiste em utilizar o sistema, já descrito, expondo-o a determinadas condições a fim de analisar tanto o funcionamento do protocolo OPC, quantos os controles P, PI e PID para o controle de nível do reservatório que possui um sensor de nível associado. Os estudos de casos propostos são os seguinte:

- Caso 1:o sistema será submetido a uma variação de referência, de forma que as bombas 1 e 2 serão utilizadas para inserir e retirar água do reservatório 1 ;

- Caso 2: o sistema será submetido tanto a variação de referência e a uma perturbação de carga através da bomba 2 que, também, atu- ará para auxiliar o controle de nível juntamente com a bomba 1. Com a observação de que a perturbação é realizada acionando a bomba 2 a $20 \%$ de sua capacidade nominal entre os segundos 20 e 30 e 50 e 60 ;

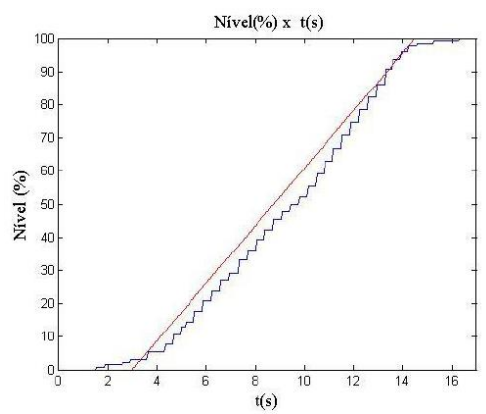

Figura 2. Resposta ao degrau do módulo de níve

Onde através da tabela 2 obtemos os seguinte parâmetros de sintonia dos controladores digitais da equação 1 expostos na tabela que se segue.

Tabela 2. Sintonia dos controladores PID

\begin{tabular}{|c|c|c|c|}
\hline Controlador & $K_{p}$ & $K_{i}$ & $K_{d}$ \\
\hline $\mathrm{P}$ & $11 / 3$ & --- & --- \\
\hline $\mathrm{PI}$ & 3,3 & 0,3634 & --- \\
\hline $\mathrm{PID}$ & 4,4 & 0,8067 & 6 \\
\hline
\end{tabular}

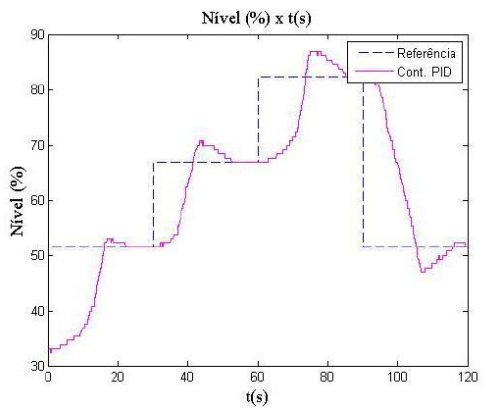

Figura 3. Controlador PID - Variação de referência - Caso 1

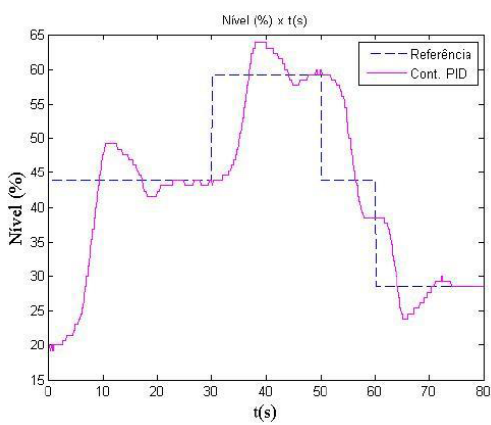

Figura 4. Controlador PID - Perturbação de Carga - Caso 2

3.2 Módulo Laboratorial 2 -Módulo de nível e Temperatura

O Módulo Laboratorial foi desenvolvido para possibilitar análise e estudo da dinâmica de variáveis 
associadas ao controle de processos industriais (Campos, 2007), tendo a escolha recaído sobre as malhas de controle de nível e temperatura, em configurações SISO e TITO (Figura 5), com características integradoras e de atraso de transporte (Wade, 2004).

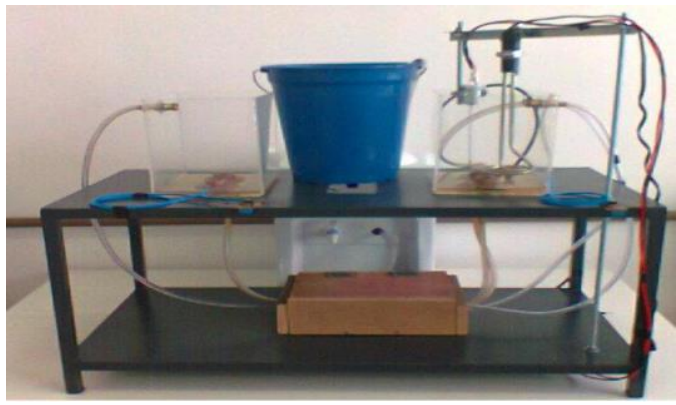

Figura 5. Módulo de Nível e Temperatura

O sistema é composto por: 2 reservatórios, 2 eletrobombas automotivas, sensores de nível e temperatura, resistor de aquecimento e um CLP, com as características:

Reservatórios: em acrílico, capacidade de 6 litros, sensores de nível e temperatura, resistor de aquecimento e interação através das eletrobombas, que circulam a água em fluxos opostos;

Eletrobombas: Denominadas bombas de esguicho ou de injeção de combustível, de baixo custo, alimentação de $12 \mathrm{~V}$, vazão nominal de 0,135 litros/s, o que propicia dinâmica satisfatória para as práticas laboratoriais;

Sensor de nível: sensor de pressão monolítico (FREESCALE SEMICONDUCTOR, 2012), modelo MPXV5004DP, com saída em tensão, 0-5V, proporcional à entrada de 0 a 40 cm H20;

Sensor de temperatura: LM35, analógico, alimentação $4-20 \mathrm{~V}$, saída de $10 \mathrm{mV} / 0 \mathrm{C}$, precisão de $0,5^{\circ} \mathrm{C}$, faixa de operação $-55^{\circ} \mathrm{C}-150{ }^{\circ} \mathrm{C}$ (NATIONAL SEMICONDUCTOR, 2012);

CLP: utilizou-se um CLP ATOS com capacidade para operar com o protocolo aberto de comunicação OPC.

Este módulo possibilita a realização de práticas laboratoriais com uma visão diferenciada. Uma primeira observação é que o módulo possibilita a análise dinâmica e o controle, em configuração SISO "Single-input and Single-ouput" - e/ou MIMO "Multiple-Input and Multiple-Output", de malhas de nível de fluidos, integrativas, e de temperatura, com grande atraso de transporte, através da utilização de um sistema supervisório; os módulos didáticos normalmente utilizados, geralmente, não apresentam a opção MIMO.

Pelas limitações de espaço, que inviabiliza apresentação mais detalhada das práticas possíveis, será apresentada uma aplicação para estudo da dinâmica de uma malha de nível com os controladores P, PI e PID digitais, utilizados tanto na indústria como na academia. O módulo possibilita a escolha, pelo usuá- rio, do procedimento a adotar: pode-se utilizar o supervisório, com estimação do modelo e dos dados de sintonia, ou através do Simulink, onde os procedimentos devem ser executados passo-a-passo.

Utilizando-se os procedimentos descritos no módulo anterior, de forma resumida são apresentados os resultados dos ensaios utilizando controladores $\mathrm{P}$, PI e PID posicionais digitais. A Resposta ao Degrau é apresentada na Figura 6, onde as características integradoras do processo de nível são evidentes:

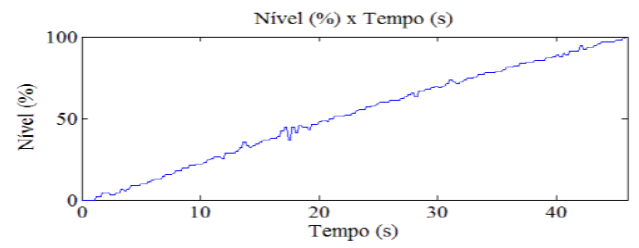

Figura 6. Resposta ao Degrau do Módulo Físico

A partir destes resultados, os parâmetros de sintonia são mostrados na Tabela 3:

Tabela 3. Sintonia dos controladores PID

\begin{tabular}{|c|c|c|c|}
\hline Controlador & $K_{p}$ & $K_{i}$ & $K_{d}$ \\
\hline $\mathrm{P}$ & 0,4525 & - & - \\
\hline $\mathrm{PI}$ & 0,4073 & 0,0111 & - \\
\hline $\mathrm{PID}$ & 0,543 & 0,0247 & 2,9867 \\
\hline
\end{tabular}

Calculados os parâmetros, pode ver o resultado do controle, para diferentes variações de referência do nível do sistema, para as três estruturas de controlador utilizadas (Figura 7, 8 e 9).

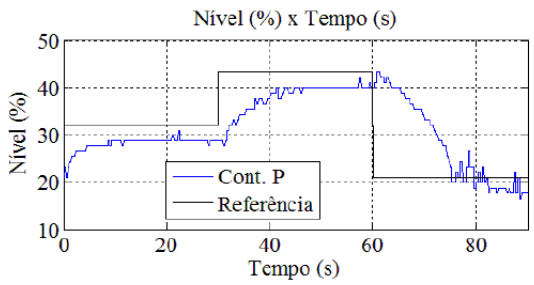

Figura 7. Controle de Nível do módulo P.

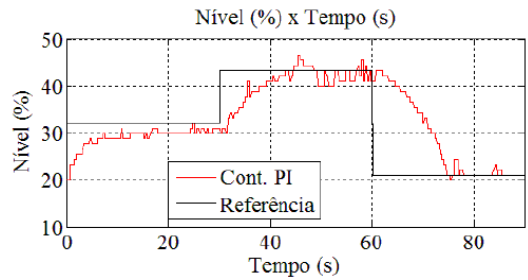

Figura 8. Controle de Nível do módulo PI.

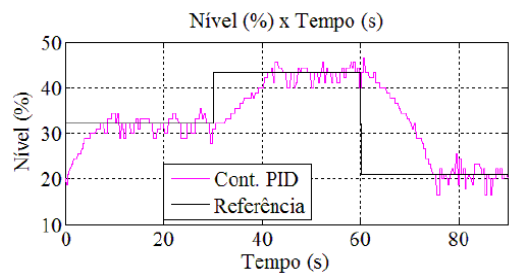

Figura 9. Controle de Nível do módulo PID. 


\subsection{Módulo Laboratorial 3}

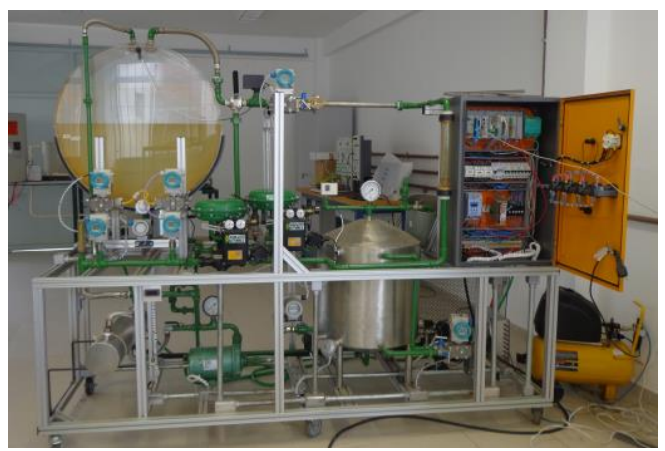

Figura 10. Módulo Laboratorial 3

O Módulo Laboratorial 3 ou Planta Didática de Processos Contínuos (Figura 10) consistiu de um sistema de tanques duplo, com aquecimento e resfriamento, que possibilita o controle das quatro variáveis de processo - vazão, nível, temperatura e pressão - em circuito único, configurado segundo topologias distintas. O sistema permite alteração das variáveis de controle e controladas, para cada malha distinta, em concepção por realimentação ("feedback") ou por antecipação ("feedforward"). Opera baseada na padronização de sinais de 4 a $20 \mathrm{~mA}$, adicionalmente ao protocolo digital Profibus PA. (Gomes, 2008). O Sistema Supervisório (figura 11) disponibiliza interface gráfica e publicação das telas gráficas dinâmicas, em tempo real.

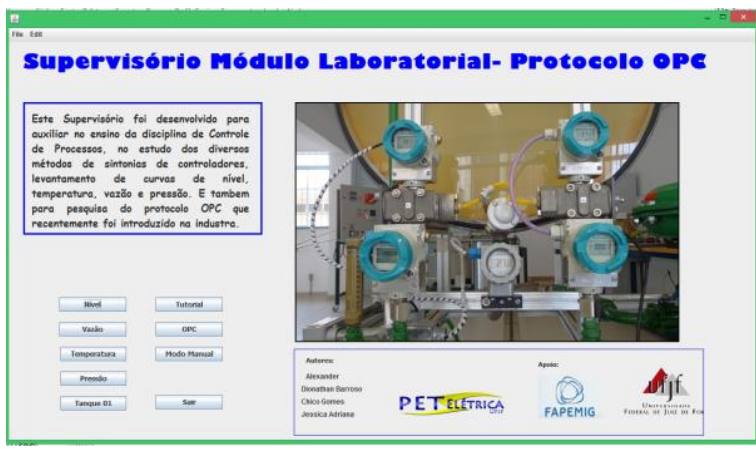

Figura 11. Supervisório JAVA “Cliente OPC”

Adicionalmente às características específicas de cada malha, o projeto do sistema prevê complexidades adicionais, comuns em ambientes industriais. Dentre estas vale citar:

- Atrasos de transporte variáveis;

- Modelagem não linear;

- Controle não linear;

- Alteração de variáveis de controle para uma mesma malha através da potência dissipada na resistência de aquecimento do tanque inferior, através da vazão do fluido, mantendo-se constante a potência da resistência de aquecimento, através da taxa de resfriamento do radiador, para vazão e potência constantes, e também para qualquer das configu- rações citadas, é possível alteração nas variáveis durante o processo.

Para se sintetizar este módulo com softwares livres (FOSS) e verificar a eficácia destes para utilização a nível industrial somado ao protocolo de comunicação OPC, foram utilizados o Scilab "cliente OPC" que trabalha na aquisição dos gráficos de respostas do sistema, na obtenção dos parâmetros de sintonia e da função de transferência. Obtido estes parâmetros o supervisório desenvolvido em linguagem de JAVA "cliente OPC" pode simular características especificas de cada malha, como no exemplo que segue.

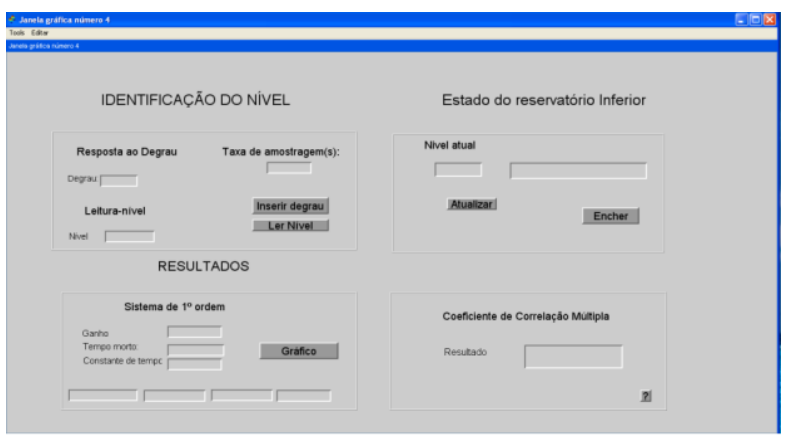

Figura 12. Tela de identificação nível - Scilab

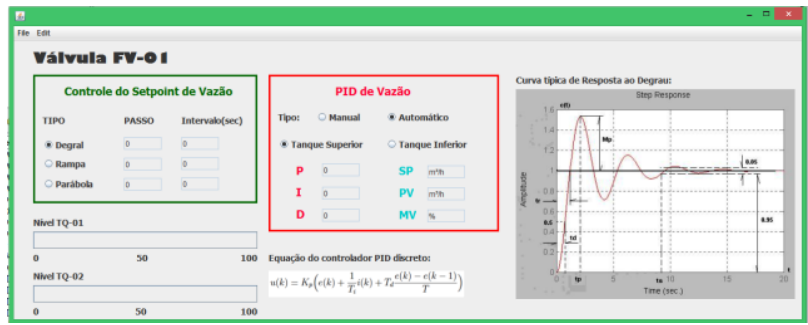

Figura 12. Controle Vazão - Supervisório Java

Este módulo ainda está sendo aprimorado, seu supervisório JAVA ainda em desenvolvimento promoverá aos alunos da Universidade Federal de Juiz de Fora (UFJF), práticas laboratoriais de forma a faze-los irem de encontro as tecnologias e protocolos de comunicação recentemente difundidos na indústria.

\section{Conclusão}

Diante dos resultados apresentados observa-se a aplicabilidade e a utilidade do sistema que mostraram-se satisfatórios, o que demonstra que a metodologia empregada desde os elementos físicos até a escolha do uso do protocolo de comunicação é válida.

No mais, podemos dizer que o trabalho obteve resultados que propiciam compreender o vasto campo de aplicação que o protocolo de comunicação OPC possui.

Um destaque importante é quanto aos usos de softwares livres no caso do Scilab e da linguagem 
JAVA, que se adequam perfeitamente ao protocolo OPC, por meio de toolbox e bibliotecas respectivamente, estes são motivadores para continuação deste trabalho, em especial ao JAVA, que possuiu um potencial muito grande para criação de ambientes interativos e interfaces gráficas.

O principal benefício de seu aprendizado e utilização consiste na redução dos custos e tempo de desenvolvimento de interfaces e drivers de comunicação, com consequente redução de custo de integração de sistemas. Esta potencialidade facilita sobremaneira que sejam escolhidos, dentro de diversas opções, os tipos de controle que melhor se adaptarão ao processo.

A soma dos três módulos estudados resultam em um acúmulo de conhecimento gradativo que tem o potencial de propiciar a qualquer profissional ou investigador o aprendizado nas mais diversas técnicas de controle, sobre o protocolo OPC, dentre outros.

Sua utilização nas atividades laboratoriais na UFJF, reforça o conhecimento técnicos dos alunos em áreas diversas, abrangendo não só os aspectos mais conceituais da teoria de controle, mas os aspectos práticos de conexão e configuração de sistemas, presentes no meio industrial e cujo conhecimento torna-se, cada vez mais, uma necessidade para os engenheiros.

\section{Agradecimentos}

Agradecemos ao Programa de Educação tutorial (PET-MEC), CAPES, FAPEMING, CNPQ e UFJF.

\section{Referências Bibliográficas}

Atos Automação Industrial. Curso básico de Controladores Programáveis, Ref.5-0026.110, Manual - Fevereiro/2006. Disponível em: http://www.atos.com.br. [Acessado em: abril de 2013]

Astrom, K.J. \& Hagglund, T. Hagglund. 1995. PID Controllers, Theory, Design and Tuning (2nd)

Campos, M. C. M. M.; Teixeira, H. C. G. Controle Típico de Equipamentos e Processos Industriais. ed. Edgard Blücher: São Paulo, 2007.

Freescale Semiconductor. Integrated Silicon Pressure Sensor On-Chip Signal Conditioned, emperature Compensated and Calibrated, 2012. Disponível em:http://www.freescale.com/files/sensors/doc/d ata_sheet/MPXV5004G.pdf. [Acessado em: 23 de abril de 2013]

Gomes, F. J. \& Pinto, D. P. Laboratórios Integrados para Controle de Processos e Análise da Eficiência Energética de Sistemas Industriais, COBENGE, 2008.

OPC Foundation, What is OPC?, 2012. Disponível em:http://www.opcfoundation.org/Default.aspx/0 1_about/01_whatis.asp?MID=AboutOPC.

[Acessado em: 21 de abril de 2013]
Santos Neto, A. F.; Barroso, D. S. Tornando a Educação em Controle de Processos mais realista: a utilização do protocolo OPC, COBENGE, 2012.

Ziegler, J. G. \& Nichols, N. B. 1942. Optimal settings for automatic controllers. Transactions of the ASME.

Wade, H. L. Basic and Advanced Regulatory Control: System Design and Application. ed. Instrumentation System, 2004. 